\title{
Antioxidant-Mediated Effects in a Gerbil Model of Iron Overload
}

\author{
Maya Otto-Duessel ${ }^{\mathrm{a}}$ Michelle Aguilar ${ }^{\mathrm{b}}$ Rex Moats ${ }^{\mathrm{a}}$ John C. Wood ${ }^{\mathrm{a}, \mathrm{b}}$ \\ Divisions of a Radiology and bediatric Cardiology, Children's Hospital Los Angeles, Los Angeles, Calif., USA
}

\section{Key Words}

Iron overload $\cdot$ Taurine $\cdot$ Heart $\cdot$ Liver $\cdot$ Antioxidants

\begin{abstract}
Introduction: Iron cardiomyopathy is a lethal complication of transfusion therapy in thalassemia major. Nutritional supplements decreasing cardiac iron uptake or toxicity would have clinical significance. Murine studies suggest taurine may prevent oxidative damage and inhibit $\mathrm{Ca}^{2+}$-channelmediated iron transport. We hypothesized that taurine supplementation would decrease cardiac iron-overloaded toxicity by decreasing cardiac iron. Vitamin $\mathrm{E}$ and selenium served as antioxidant control. Methods: Animals were divided into control, iron, taurine, and vitamin E/selenium groups. Following sacrifice, iron and selenium measurements, histology, and biochemical analyses were performed. Results: No significant differences were found in heart and liver iron content between treatment groups, except for higher hepatic dry-weight iron concentrations in taurine-treated animals ( $p<0.03$ ). Serum iron increased with iron loading (751 \pm 66 vs. $251 \pm 54 \mu \mathrm{g} / \mathrm{dl}, \mathrm{p}<0.001)$ and with taurine (903 \pm $136 \mu \mathrm{g} / \mathrm{dl}, \mathrm{p}=0.03)$. Conclusion: Consistent with oxidative stress, iron overload increased cardiac malondialdehyde levels, decreased heart glutathione peroxidase (GPx) activity, and increased serum aspartate aminotransferase. Taurine
\end{abstract}

ameliorated these changes, but only significantly for liver GPx activity. Selenium and vitamin E supplementation did not improve oxidative markers and worsened cardiac GPx activity. These results suggest that taurine acts primarily as an antioxidant rather than inhibiting iron uptake. Future studies should illuminate the complexity of these results.

Copyright $\odot 2007$ S. Karger AG, Basel

\section{Introduction}

Antioxidants appear to play an important role in the improvement of iron-mediated heart diseases. Friedreich's ataxia, for example, is an inherited neurological disease that is associated with iron overload in the mitochondria and oxidative damage, which ultimately can result in cardiomyopathy. Recently, it has been reported that treatment with the antioxidants idebenone, coenzyme $\mathrm{Q}_{10}$, or vitamin E leads to improvement of cardiomyopathy in patients that suffer from this disorder [1-3]. Oxidative stress due to iron overload has also been observed in people that suffer from thalassemia or sickle cell disease. Whereas sickle cell patients displayed more signs of inflammation, oxidative stress is more pronounced in thalassemics [4].

Dr. John Wood

Division of Cardiology, Mail Stop 34

Children's Hospital of Los Angeles

4650 Sunset Blvd, Los Angeles, CA 90027 (USA)

Tel. +1 323669 5470, Fax +1 323669 731, E-Mail jwood@chla.usc.edu 
Thalassemia patients develop cardiac iron overload toxicity as a complication of monthly hypertransfusion therapy. Although iron chelation therapy greatly decreases iron burden, those patients still remain at risk to develop iron-mediated toxicity and iron overload [4]. Medications or nutritional supplements that could potentially reduce iron load and iron-induced cardiovascular injury would have clinical significance. Present in high concentrations in both human and rodent myocytes, the amino acid taurine demonstrates antioxidant properties, and plays a role in modulating intracellular $\mathrm{Ca}^{2+}$ levels [5-8]. In mice, taurine supplementation appears to decrease iron-induced cardiac toxicity [5]. Its mechanism is still not fully understood; it has been speculated that taurine acts as an antioxidant or inhibits iron uptake via calcium channels $[5,9,10]$. Supplementation with vitamin $\mathrm{E}$ and selenium has also been shown to protect against oxidative damage and reduce iron concentrations in iron-overloaded mice [11]. It has been known that thalassemia patients are deficient in selenium, therefore selenium supplementation might be beneficial [12].

The Mongolian gerbil exhibits a similar pathophysiology to that observed in humans in response to iron overload [13-16]. The purpose of this study was to try to differentiate taurine's potential role in preventing myocardial iron uptake versus its role as an antioxidant in the gerbil model. Treatment with vitamin $\mathrm{E}$ and selenium was chosen to model the effects of antioxidant therapy alone. We hypothesized that supplementation with taurine would concomitantly decrease iron levels and ironinduced oxidative damage to the heart and liver.

\section{Methods}

Animals and Experimental Design

This investigation was approved by the Institutional Animal Care and Use Committee of the Children's Hospital Los Angeles. Twenty-six 8- to 10-week-old female Mongolian gerbils, obtained from Charles River Laboratories, were divided into four groups. Eight animals served as age-matched controls and did not undergo iron loading or treatment. The iron-only group $(n=8)$ received weekly subcutaneous injections of iron dextran at a dose of $200 \mathrm{mg} / \mathrm{kg} /$ week over a period of 10 weeks, but did not receive any supplementation. Taurine $(\mathrm{n}=5)$ or selenium and vitamin $\mathrm{E}$ $(\mathrm{n}=5)$ supplementation began 2 weeks prior to initiating the 10 week iron overload period and continued throughout the 10 weeks of iron injections. Taurine $(12.5 \mathrm{~g} / \mathrm{l})$ and selenium $[1 \mathrm{ppm}$ (sodium selenate $1.67 \mathrm{~g} / \mathrm{l})$, dissolved in $10 \%$ dextrose and water solution] were administered via drinking water. Vitamin E ( $\alpha$-tocopherol acetate) supplementation was administered subcutaneously at a dose of $400 \mathrm{mg} / \mathrm{gerbil} /$ day. All compounds were purchased from Sigma (St. Louis, Mo., USA). Following the 2-week pretreatment period, 24 animals received iron dextran loading at a dose of 200 $\mathrm{mg} / \mathrm{kg}$ body weight for 10 weeks followed by a 13-day iron equilibration period.

\section{Biochemical Analysis}

Animals were injected intraperitoneally with ketamine/xylazine $(50 \mathrm{mg} / \mathrm{kg} / 5 \mathrm{mg} / \mathrm{kg}$ ); blood was drawn directly from the hearts, and all organs were perfused. For the perfusion procedure, hearts were exposed by incisions along the rib cage and diaphragm and the left ventricle was pierced with a $30 \mathrm{~g}$ syringe needle. Ten milliliters of PBS were slowly injected into the left ventricle with a $10-\mathrm{ml}$ syringe. A small incision was made in the right atrium to release returned circulation. Serum was sent for aspartate aminotransferase (AST), alanine transaminase (ALT), and serum iron level determination to the Children's Hospital Los Angeles clinical laboratory.

Lipid peroxidation was determined by the measurement of malondialdehyde (MDA) using a lipid hydroperoxidation assay kit (Calbiochem, La Jolla, Calif., USA). In brief, sections of the perfused heart and liver were immediately homogenized upon collection and lipid hydroperoxidases were extracted according to the company's protocol. Standard MDA was prepared by hydrolysis of 13-hydroperoxyoctanoic acid. Absorbance was determined at $500 \mathrm{~nm}$ using a $1-\mathrm{ml}$ cuvette and a spectrophotometer (Spectronic GENESYS 8, Thermo Fisher Scientific, Waltham, Mass., USA).

For measuring glutathione peroxidase (GPx) activity, we followed the manufacturer's instructions (Calbiochem, La Jolla, Calif., USA). Following perfusion of heart and liver, tissue samples were collected, homogenized ( $50 \mathrm{~mm}$ Tris- $\mathrm{HCl}, \mathrm{pH} 7.5,5 \mathrm{~mm}$ EDTA and $1 \mathrm{mM}$ DDT) and centrifuged $(10,000 \mathrm{~g}$ for $15 \mathrm{~min}$ at $4^{\circ} \mathrm{C}$ ). Following mixing of cumene hydroperoxide with supernatant, absorbance at $340 \mathrm{~nm}$ was measured immediately and read every minute over $7 \mathrm{~min}$. The decrease in absorbance at $340 \mathrm{~nm}$, which is caused by the oxidation of NADPH into $\mathrm{NADP}^{+}$, is directly proportional to the GPx activity. One unit of activity is defined as the amount of enzyme that catalyzes the oxidation of $1 \mathrm{nmol} \mathrm{NADPH} / \mathrm{min}$, with a molar coefficient of extinction of $6.22 \times 10^{6} \mathrm{M}^{-1} \mathrm{~cm}^{-1}$ used for NADPH.

\section{Selenium and Iron Assessment}

Perfused hearts and livers were weighed wet, and sections sent to Mayo Medical Laboratories (Rochester, Minn., USA), where total ('nonheme') iron was assayed and tissue dry weight and dryweight iron concentrations were reported. Heart and liver samples were also sent to Galbraith Laboratories (Knoxville, Tenn., USA), where dry-weight selenium concentrations were determined by graphite furnace atomic absorption.

\section{Histology}

The remaining portions of these organs were immersion fixed in $10 \%$ formalin, paraffin embedded and stained with hematoxylin and eosin, Masson's trichrome, and Prussian blue.

Statistical Analysis

Statistical significance of the data was assessed with the Student $t$ test, with $p<0.05$ considered significant. All data are presented as means \pm standard deviation. 
Table 1. Cardiac and hepatic organ iron contents, dry-weight iron concentrations, wet-to-dry weight ratio and organ weights

\begin{tabular}{|c|c|c|c|c|}
\hline & $\begin{array}{l}\text { Control } \\
(\mathrm{n}=8)\end{array}$ & $\begin{array}{l}\text { Iron } \\
(\mathrm{n}=8)\end{array}$ & $\begin{array}{l}\text { Taurine } \\
(\mathrm{n}=5)\end{array}$ & $\begin{array}{l}\text { Vitamin E/selenium } \\
(\mathrm{n}=5)\end{array}$ \\
\hline \multicolumn{5}{|l|}{ Heart } \\
\hline Organ weight, mg & $318.6 \pm 48.2$ & $327.8 \pm 18.8$ & $320.0 \pm 20.9$ & $362.6 \pm 49.0$ \\
\hline Wet-to-dry ratio & $5.36 \pm 0.37$ & $5.22 \pm 0.29$ & $4.94 \pm 0.30$ & $5.00 \pm 0.28$ \\
\hline$[\mathrm{Fe}]$ dry weight, mg/g & $0.36 \pm 0.02^{*}$ & $3.00 \pm 0.20$ & $3.30 \pm 0.82$ & $2.89 \pm 0.66$ \\
\hline Organ iron, mg & $0.021 \pm 0.003^{*}$ & $0.188 \pm 0.018$ & $0.213 \pm 0.050$ & $0.208 \pm 0.048$ \\
\hline \multicolumn{5}{|l|}{ Liver } \\
\hline Organ weight, g & $2.37 \pm 0.23^{*}$ & $4.43 \pm 0.28$ & $4.17 \pm 0.52$ & $5.03 \pm 1.30$ \\
\hline Wet-to-dry ratio & $4.59 \pm 0.67^{*}$ & $3.87 \pm 0.22$ & $3.87 \pm 0.25$ & $4.34 \pm 0.44^{*}$ \\
\hline$[\mathrm{Fe}]$ dry weight, $\mathrm{mg} / \mathrm{g}$ & $0.68 \pm 0.15^{*}$ & $35.8 \pm 2.8$ & $41.9 \pm 4.3^{*}$ & $36.0 \pm 6.6$ \\
\hline Organ iron, $\mathrm{mg}$ & $0.36 \pm 0.09^{*}$ & $41.1 \pm 3.5$ & $44.9 \pm 3.8$ & $40.7 \pm 7.7$ \\
\hline
\end{tabular}

Table 2. Liver enzymes and serum iron

\begin{tabular}{lllll}
\hline & Control & Iron & Taurine & Vitamin E/selenium \\
\hline Serum iron, $\mu \mathrm{g} / \mathrm{dl}$ & $250.8 \pm 54.2$ & $750.6 \pm 66.0^{*}$ & $902.6 \pm 136.0^{*+}$ & $720.8 \pm 81.8^{*}$ \\
AST, U/l & $457.5 \pm 129.5$ & $566.4 \pm 80.5$ & $612.0 \pm 289.8$ & $380.2 \pm 215.4$ \\
ALT, U/l & $112.8 \pm 48.2$ & $122.8 \pm 39.0$ & $120.4 \pm 71.4$ & $99.6 \pm 75.8^{+}$ \\
\hline
\end{tabular}

${ }^{*} \mathrm{p}<0.05$ vs. control; ${ }^{+} \mathrm{p}<0.05$ vs. iron.

\section{Results}

Cardiac and liver organ iron contents, dry-weight iron concentrations, wet-to-dryweight ratio and organ weights are summarized in table 1 . Hearts and livers from ironloaded animals demonstrated 9- to 115-fold higher organ iron content than controls. Iron-loaded livers also exhibited greater total organ weight and lower wet-to-dry weight ratio than livers from control animals. Taurinetreated animals demonstrated greater dry-weight liver iron concentrations (41.9 vs. $35.8 \mathrm{mg} / \mathrm{g}$ ) but total liver iron content was not different. No other differences were observed in heart and liver iron concentration or content between the three treatment groups.

Liver enzymes and serum iron are summarized in table 2. Serum iron was tightly correlated $\left(\mathrm{r}^{2}=0.92\right)$ with wet-weight liver iron concentration (not shown). Serum iron was significantly elevated in all iron-loaded animals, but particularly the taurine group. AST and ALT were correlated with one another $\left(\mathrm{r}^{2}=0.7\right)$ but not with serum or hepatic iron concentration. AST trended higher in the iron-alone and taurine-treated groups but not in the vitamin E/selenium-treated animals. ALT was unchanged across treatment groups.

Figure 1 depicts cardiac and hepatic iron staining with Prussian blue for each treatment group after 10 weeks of iron loading. Cardiac iron demonstrates intense staining in interstitially distributed endothelial cells and more diffuse myocyte staining as previously described [17, 18]. In the liver, iron-treated animals showed iron distribution in hepatocytes, Kupffer cells, and large aggregates of phagocytic cells. There was no visible change in iron distribution between the three groups for either liver or heart.

MDA levels of heart and liver are shown in figure 2. In the heart, iron loading led to significantly higher MDA levels, reflecting increased oxidative damage. MDA levels in taurine-treated animals were $11 \%$ lower compared to the iron-only group, but this difference did not reach statistical significance. There was no beneficial effect of vitamin E/selenium. 


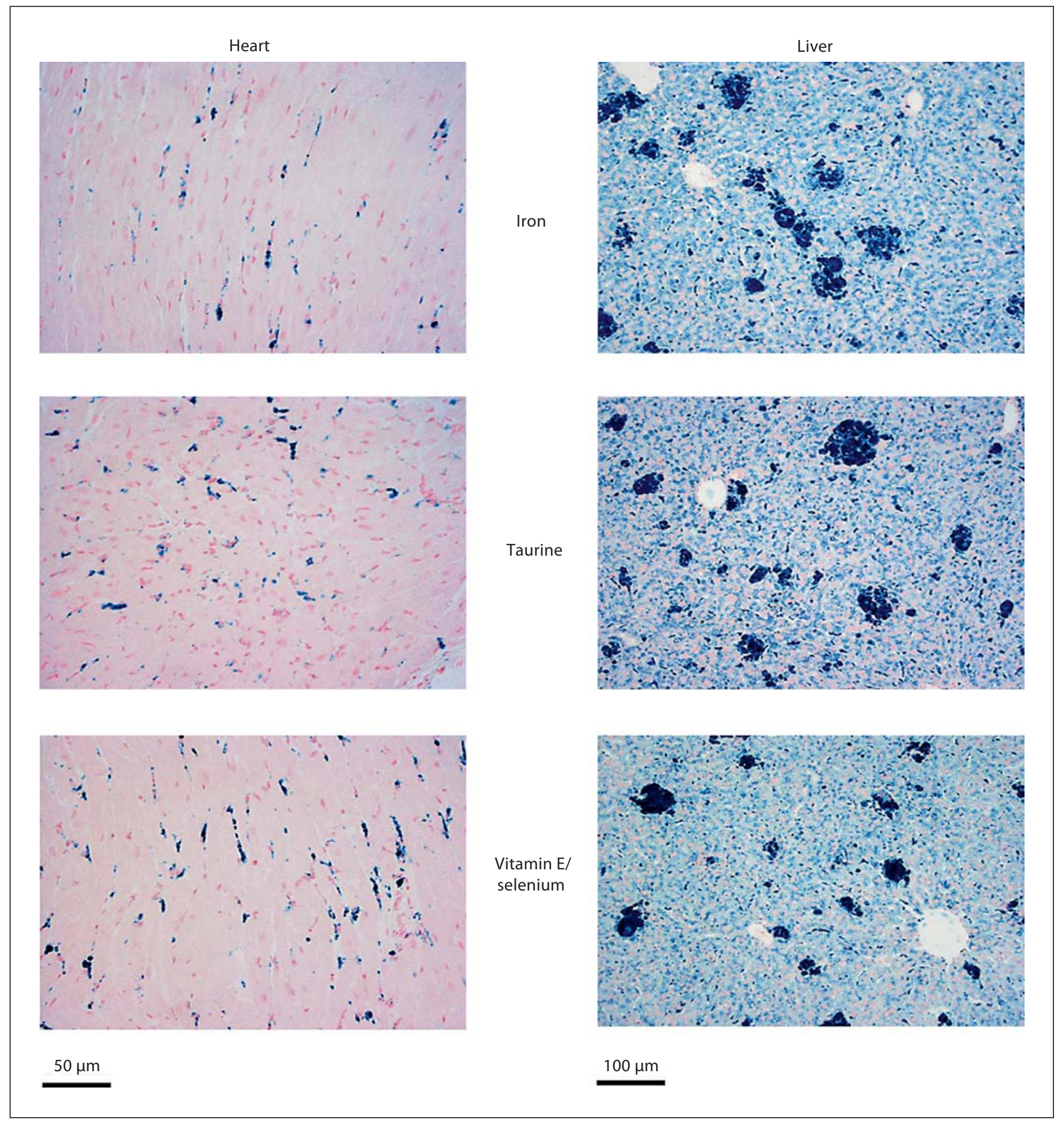

Fig. 1. Histological analysis of heart and liver after 10 weeks of iron loading. Groups were stained with Prussian blue iron stain. No fundamental changes in hepatic and cardiac iron distribution were observed between the three groups. 

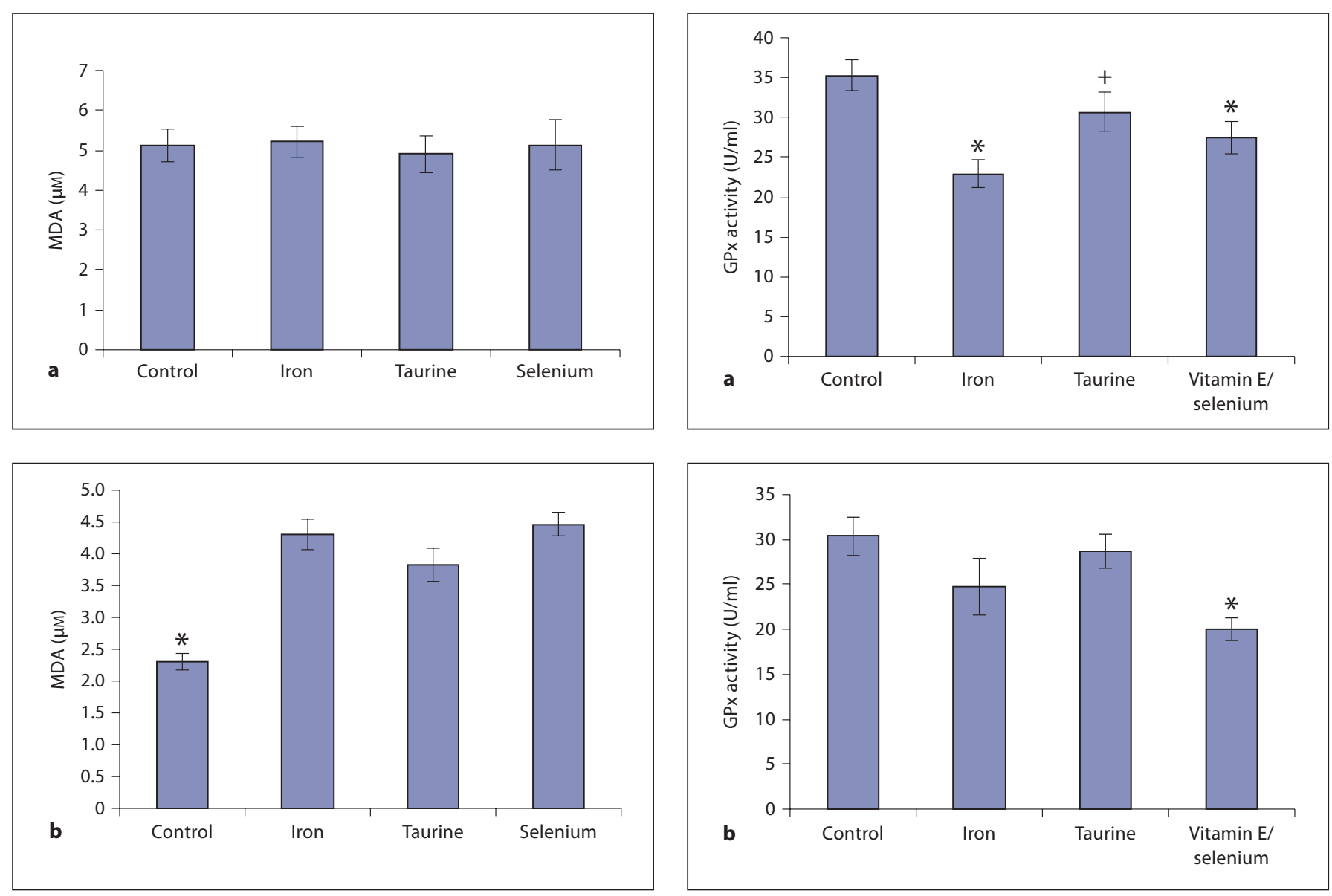

Fig. 2. MDA levels in liver (a) and heart (b). a Iron overload in the liver did not cause MDA levels to rise significantly compared to hepatic MDA levels of unloaded gerbils (control). b Cardiac MDA levels were significantly elevated in all three treatment groups compared to control. MDA levels in taurine-treated animals trended lower (11\%) than MDA levels in iron-only animals. No significant difference was detected between cardiac MDA levels in vitamin E/selenium-treated animals compared to MDA levels of iron-only animals. ${ }^{*} \mathrm{p}<0.05$ vs. iron.

No significant differences were detected in liver MDA levels between the iron group (fig. 2a) and the control group suggesting that iron overload did not increase MDA production in the liver. As a result, no treatment differences could be observed.

Figure 3 demonstrates liver and heart GPx activity levels. Iron loading without antioxidants reduced GPx activity by $35 \%$. GPx activity was spared in taurine-treated animals (13\% less than controls, $\mathrm{p}=$ nonsignificant). Vitamin E/selenium produced intermediate results with GPx activity $22 \%$ less than controls.

Fig. 3. Measurement of GPx activity levels in liver (a) and heart (b). a Significantly reduced hepatic GPx activity in iron-only and vitamin E/selenium animals was observed in comparison to control. Taurine seemed to have a protective effect on GPx activity. Only a small decrease (13\%) of GPx activity was noted compared to control. There was a significant difference between GPx activity in taurine-treated animals versus iron-only animals. b In the heart, GPx activity showed a similar trend. ${ }^{*} \mathrm{p}<0.05$ vs. control; ${ }^{+} \mathrm{p}<0.05$ vs. iron.

GPx activity in the heart was qualitatively similar but changes did not reach statistical significance. Iron loading produced $18.8 \%$ lower GPx activity; this deficit was only $10 \%$ with concomitant taurine treatment. Surprisingly, vitamin E/selenium supplementation lowered cardiac GPx activity $37.5 \%$ with respect to control, indicating a detrimental effect of this therapy in the heart.

Selenium concentrations in the heart and liver are plotted in figure 4. Iron loading reduced selenium levels in both organs by 34 and $47 \%$, respectively; however, interanimal variability was sufficiently high that these changes did not reach statistical significance. Only the

Acta Haematol 2007;118:193-199 

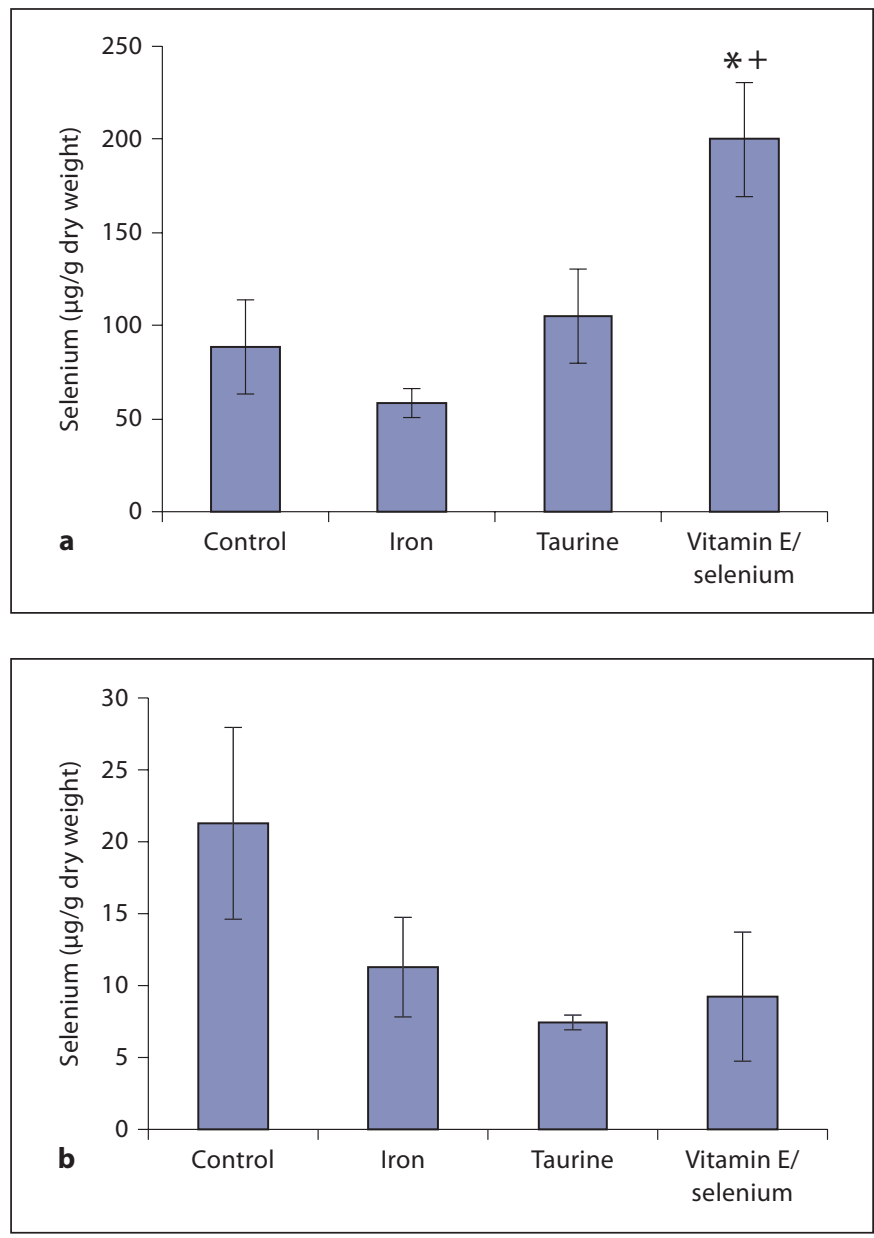

Fig. 4. Depiction of selenium concentrations in heart (a) and liver (b). Selenium levels were reduced in iron-loaded animals compared to controls, although this was not statistically significant. Significantly elevated levels of selenium were only found in the hearts of vitamin E/selenium-supplemented animals. ${ }^{*} \mathrm{p}<0.05$ vs. control; ${ }^{+} \mathrm{p}<0.05$ vs. iron.

vitamin E/selenium-supplemented group demonstrated significantly elevated heart selenium levels ( $127 \%$ of control), while no significant differences were noted in the other groups or the liver (fig. 4a, b).

\section{Discussion}

Prior murine work suggested selective cardioprotective effects of taurine mediated, in part, through decreased cardiac iron levels. In the present work, taurine improved surrogates of oxidative stress in the liver and the heart, despite increasing liver iron concentration and serum iron. These findings indicate that taurine acts pri- marily as an antioxidant rather than by decreasing tissue iron burden. Alternatively, taurine could be decreasing the lability of intracellular iron either by direct association or by upregulating intracellular ferritin production; these questions would best be addressed by fluorescent markers and myocyte culture [19].

The increase in hepatocyte and serum iron concentrations by taurine therapy is an unexpected finding. Subtherapeutic iron chelation therapy can produce similar results [18]. The mechanism is unknown but likely represents interference with spontaneous iron elimination. Gerbils excrete iron predominantly through the feces, suggesting a biliary mechanism. Taurine may impede this process, leading to higher iron levels than in untreated animals. The serum iron was highly correlated with liver iron.

Although selenium supplementation successfully raised heart selenium levels, it lowered cardiac GPx levels relative to untreated animals, suggesting that low selenium may be a marker of oxidant stress, rather than a cause. The trend toward higher cardiac selenium values in taurine-treated animals supports this hypothesis. The mechanism of the vitamin E/selenium toxicity is unclear and counter to the findings observed in mice [11]. Since selenium levels were raised to a level more than double control values, selenium dosage may have been inappropriately high. However, liver selenium levels were unchanged by supplementation, suggesting tissue specificity in selenium level regulation. Thus while iron overload is associated with low serum selenium levels in patients, indiscriminant supplementation may be inadvisable.

Our study has several possible limitations. First, our sample size was chosen based upon pilot data that suggested a larger effect size. As a result, some of the antioxidative surrogate comparisons may have been underpowered to detect significant differences. Future studies involving either a more aggressive iron loading scheme, to enhance organ toxicity, or larger group sizes could be beneficial. Nonetheless, in our present work we were able to clearly demonstrate that taurine's primary benefit was not a decrease in tissue iron.

A second limitation is that cardiac iron storage represents a mixture of myocyte and endomysial iron storage in rodents. Endomysial iron storage occurs early during iron loading with secondary redistribution to the myocyte and functional abnormalities [17]. The MDA and GPx assays cannot distinguish contributions from the different pools, although myocytes contribute overwhelmingly on a volumetric basis. An analogous situation exists in the liver with hepatocytes and the re- 
ticuloendothelial system. Thus identifying redox damage in a specific tissue is not possible with present techniques.

The failure of liver MDA to increase with iron loading is puzzling, particularly with the intuitive results observed in the heart. Although MDA is an accepted marker for lipid peroxidation, the MDA assay has its limitations, which may be relevant to our results [4]

\section{Conclusion}

Taurine supplementation partially ameliorated ironinduced toxicity in the iron-loaded gerbil without decreasing tissue iron burden, with larger effects observed in the liver than the heart. Further work is necessary to determine the mechanisms of liver protection and modulation of iron transport. Coadministration of vitamin $\mathrm{E}$ and selenium was ineffective in the liver and even detrimental in the heart in this model.

\section{References}

1 Cooper JM, Schapira AH: Friedreich's ataxia: disease mechanisms, antioxidant and coenzyme Q10 therapy. Biofactors 2003;18: 163-171.

$\checkmark 2$ Hausse A: Idebenone and reduced cardiac hypertrophy in Friedreich's ataxia. Heart 2002;87:316-317.

3 Kadar K: Successful idebenone therapy of hypertrophic cardiomyopathy in Friedreich ataxia. Orv Hetil 2003;144:1241-1244.

$\checkmark 4$ Walter PB, Fung EB, Killilea DW, Jiang Q, Hudes M, Madden J, Porter J, Evans P, Vichinsky E, Harmatz P: Oxidative stress and inflammation in iron-overloaded patients with beta-thalassaemia or sickle cell disease. Br J Haematol 2006; 135:254-263.

5 Oudit GY, Trivieri MG, Khaper N, Husain T, Wilson GJ, Liu P, Sole MJ, Backx PH: Taurine supplementation reduces oxidative stress and improves cardiovascular function in an iron-overload murine model. Circulation 2004;109:1877-1885

-6 Schaffer SW, Kramer J, Chovan JP: Regulation of calcium homeostasis in the heart by taurine. Fed Proc 1980;39:2691-2694.

7 Claire H: Acute effects on taurine on intracellular calcium in normal and diabetic cardiac myocytes. Eur J Physiol 1999;438:384391.
8 McBroom MJ, Welty DJ: Effects of taurine and verapamil on heart calcium in hamsters and rats. Comp Biochem Physol 1985; 82C:279-281.

$\checkmark 9$ Oudit GY, Sun H, Trivieri MG: L-type Ca(2+) channels provide a major pathway for iron entry into cardiomyocytes in iron-overload cardiomyopathy. Nat Med 2003;9:11871194.

10 Satoh H, Horie M: Actions of taurine on the L-type $\mathrm{Ca}^{2+}$ channel current in guinea pig ventricular cardiomyocytes. J Cardiovasc Pharmacol 1997;30:711-716.

11 Bartfay WJ, Hou D, Brittenham Gl: The synergistic effects of vitamin $\mathrm{E}$ and selenium in iron-overloaded mouse hearts. Can J Cardiol 1998;14:937-941.

12 Bartfay WJ, Bartfay E: Selenium and glutathione peroxidase with beta-thalassemia major. Nurs Res 2001;50:178-183.

13 Hershko C, Link G, Konijn AM, Huerta M, Rosenmann E, Reinus C: The iron-loaded gerbil model revisited: effects of deferoxamine and deferiprone treatment. J Lab Clin Med 2002;139:50-58.
14 Carthew P, Dorman BM, Edwards RE, Francis JE, Smith AG: A unique rodent model for both the cardiotoxic and hepatotoxic effects of prolonged iron overload. Lab Invest 1993; 69:217-222.

5 Obejero-Paz CA, Yang T, Dong WQ: Deferoxamine promotes survival and prevents electrocardiographic abnormalities in the gerbil model of iron-overload cardiomyopathy. J Lab Clin Med 2003;141:121-130.

16 Yang T, Dong WQ, Kuryshev YA: Bimodal cardiac dysfunction in an animal model of iron overload. J Lab Clin Med 2002;140:263271.

17 Wood J, Otto-Duessel M, Aguilar M, Nick H, Moats R: Deferasirox and deferiprone remove cardiac iron in the iron-overloaded gerbil. Transl Res 2006;148:272-280.

18 Wood JC, Otto-Duessel M, Aguilar M, Nick $\mathrm{H}$, Coates TD, Moats R: Dose response of deferoxamine, deferiprone, and ICL670 chelation therapy in a gerbil model of iron overload. Blood 2004; 104:985a.

19 Glickstein H, El RB, Link G, Breuer W, Konijn AM, Hershko C, Nick H, Cabantchik ZI: Action of chelators in iron-loaded cardiac cells: accessibility to intracellular labile iron and functional consequences. Blood 2006; 108:3195-3203. 